\title{
Mental Health and Asthma Related Outcomes for Pediatric Patients Receiving Dupilumab Therapy
}

Saad Kamran ${ }^{1}$, Nadia Krupp ${ }^{2}$

${ }^{1}$ Indiana University School of Medicine; ${ }^{2}$ Indiana University School of Medicine, Department of Pediatrics

Background and Hypothesis: Asthma can hinder the activities that pediatric patients can do in their daily lives. This could affect their mental health because they may have anxiety about when their next asthma attack is going to occur or whether or not they will be able to participate in the activities that all their classmates are able to. In order to help reduce the detriments of asthma on mental health, pediatric patients with asthma were given Dupilumab in order to see whether their asthma and mental health outcomes improved by reducing the severity of their asthma. We hypothesized that patients using Dupilumab were going to see better outcomes in their mental health by decreasing anxiety and depression while also improving their breathing by improving their FEV1, FEV1/FVC ratio, and Asthma Control Score.

Project Methods: Pediatric patients with severe persistent asthma were entered into a High Risk Asthma clinic where they received treatment. The patients would take a spirometry test each visit and also answer questions in the GAD-7 and PHQ-9 mental health screens. The values were noted in their medical charts and also entered into a database. Using the database, the averages of all the patients' scores before they began Dupilumab and after they began Dupilumab were calculated along with the standard deviation in order to determine a statistically significant difference.

Results: Upon averaging the data, each category showed better outcomes after Dupilumab therapy, but the standard deviation always overlapped with the results from before Dupilumab therapy was started.

Conclusion and Potential Impact: Despite not being statistically significant, Dupilumab did improve the asthma and mental health outcomes of patients. Further exploration with more data in order to eliminate outlying values could change the results. Dupilumab and other biologic treatments could potentially improve asthma outcomes in the future for pediatric patients. 